\title{
First Cumulant of the Dynamic Structure Factor for Polymers in $\Theta$ Solvents. Effects of Chain Stiffness and Local Chain Conformation
}

\author{
Masashi OsA, Hiroshi UedA, Takenao YoshiZAKI, ${ }^{\dagger}$ and Hiromi YAMAKAwA \\ Department of Polymer Chemistry, Graduate School of Engineering, Kyoto University, \\ Katsura, Nishikyo-ku, Kyoto 615-8510, Japan
}

(Received August 10, 2005; Accepted October 5, 2005; Published February 15, 2006)

\begin{abstract}
The first cumulant $\Omega(k)$ of the dynamic structure factor as a function of the magnitude $k$ of the scattering vector was determined from dynamic light scattering (DLS) measurements for two atactic poly $(\alpha$-methylstyrene) (a-P $\alpha$ MS) samples with weight-average molecular weights $M_{\mathrm{w}}=3.22 \times 10^{6}$ and $5.46 \times 10^{6}$ in cyclohexane at $30.5^{\circ} \mathrm{C}$ $(\Theta)$ and for one polyisobutylene (PIB) sample with $M_{\mathrm{w}}=6.63 \times 10^{6}$ in isoamyl isovalerate at $25.0^{\circ} \mathrm{C}(\Theta)$. The translational diffusion coefficient was also determined from DLS measurements for the a-P $\alpha$ MS sample with the larger $M_{\mathrm{w}}$ and for the PIB sample in the same solvent conditions. The mean-square radius of gyration $\left\langle S^{2}\right\rangle$ was determined from static light scattering measurements for the PIB sample. From a comparison of the present data for a-P $\alpha$ MS and PIB along with the previous ones for atactic polystyrene and poly ( $n$-hexyl isocyanate) with the helical wormlike chain theory, it may be concluded that the universality predicted by the Gaussian chain theory for the plot of $\eta_{0} \Omega(k) / k_{\mathrm{B}} T k^{3}$ against $\left\langle S^{2}\right\rangle^{1 / 2} k$ cannot be realized for flexible polymers even with very high $M_{\mathrm{w}}\left(\simeq 10^{6}-10^{7}\right)$, and that the height of the plateau in the $k^{3}$-region depends on chain stiffness and local chain conformation, where $\eta_{0}$ is the solvent viscosity, $k_{\mathrm{B}}$ the Boltzmann constant, and $T$ the absolute temperature. [DOI 10.1295/polymj.38.153]

KEY WORDS Poly $(\alpha$-methylstyrene) / Polyisobutylene / Dynamic Light Scattering / Dynamic Structure Factor / First Cumulant / Helical Wormlike Chain /
\end{abstract}

Recently, experimental studies have been made of the behavior of the first cumulant $\Omega(k)$ of the dynamic structure factor as a function of the magnitude $k$ of the scattering vector for both flexible and semiflexible polymers in dilute solution. ${ }^{1,2}$ For typical flexible polymers such as atactic polystyrene (a-PS) and atactic poly(methyl methacrylate) (a-PMMA) with very high molecular weights in the respective $\Theta$ solvents, cyclohexane at $34.5^{\circ} \mathrm{C}$ and acetonitrile at $44.0^{\circ} \mathrm{C},{ }^{1}$ it has been found that the so-called universal plots of $\eta_{0} \Omega(k) / k_{\mathrm{B}} T k^{3}$ against $\left\langle S^{2}\right\rangle^{1 / 2} k$ for these polymers are appreciably, although slightly, deviated from each other in the range of large $k$, where $\eta_{0}$ is the solvent viscosity, $k_{\mathrm{B}}$ the Boltzmann constant, $T$ the absolute temperature, and $\left\langle S^{2}\right\rangle$ the mean-square radius of gyration. For semiflexible polymers, for example, $\operatorname{poly}(n-$ hexyl isocyanate) (PHIC) in $n$-hexane at $25.0{ }^{\circ} \mathrm{C},{ }^{2}$ on the other hand, it has been found that the behavior of the plot depends remarkably on chain stiffness. Such behavior of these experimental results have been shown to be consistently explained by the helical wormlike (HW) chain theory, ${ }^{3,4}$ leading to the conclusion that the plot depends on chain stiffness and local chain conformation in contradiction to the prediction by the Gaussian chain theory. ${ }^{5-7}$ In this paper, we further make a similar study of $\Omega(k)$ for atactic $\operatorname{poly}(\alpha-$ methylstyrene) (a-P $\alpha \mathrm{MS}$ ) and polyisobutylene (PIB) in $\Theta$ solvents in order to confirm the previous conclusion.

It is convenient to make here a remark on the conformational characteristics of the present two polymer chains which have been found from an analysis of their dilute solution properties on the basis of the HW chain model. ${ }^{3,8,9}$ The value of the static stiffness parameter $\lambda^{-1}$ for the a-P $\alpha$ MS chain is $46.8 \AA$, which is more than two times as large as the value $20.6 \AA$ for a-PS, and moreover, it tends to retain as large helical portions in dilute solution as the a-PMMA chain. On the other hand, the value of $\lambda^{-1}$ for the PIB chain is $15.3 \AA$, which is the smallest of the ones for all the polymers we have investigated so far. We may therefore examine effects of chain stiffness and local chain conformation on the behavior of $\Omega(k)$ for a variety of flexible polymers.

\section{EXPERIMENTAL}

\section{Materials}

The two a-P $\alpha$ MS samples AMS320 and AMS550 used in this work are the same as those used in previous studies of the gyration-, viscosity-, and hydrodynamic-radius expansion factors ${ }^{10,11}$ and the second and third virial coefficients, ${ }^{12,13}$ i.e., fractions separated by fractional precipitation from the original sam-

${ }^{\dagger}$ To whom correspondence should be addressed (E-mail: yoshizaki@molsci.polym.kyoto-u.ac.jp). 
Table I. Values of $M_{\mathrm{w}}, M_{\mathrm{w}} / M_{\mathrm{n}},\left\langle S^{2}\right\rangle^{1 / 2}$ and $R_{\mathrm{H}}$ for poly $(\alpha$-methylstyrene $)$ and polyisobutylene

\begin{tabular}{|c|c|c|c|c|}
\hline Sample & $M_{\mathrm{w}}$ & $M_{\mathrm{w}} / M_{\mathrm{n}}$ & $\begin{array}{c}10^{-2}\left\langle S^{2}\right\rangle^{1 / 2} \\
(\AA)\end{array}$ & $\begin{array}{c}10^{-2} R_{\mathrm{H}} \\
(\AA)\end{array}$ \\
\hline \multicolumn{5}{|c|}{ a-P $\alpha$ MS in Cyclohexane at $30.5^{\circ} \mathrm{C}(\Theta)$} \\
\hline AMS320a & $3.22 \times 10^{6}\left(3.24 \times 10^{6}\right)^{\mathrm{b}}$ & 1.05 & $4.6_{5}$ & $3.6_{8}$ \\
\hline AMS550 & $5.46 \times 10^{6}\left(5.56 \times 10^{6}\right)$ & & $6.0_{9}$ & $4.7_{4}$ \\
\hline \multicolumn{5}{|c|}{$\mathrm{PIB}$ in IAIV at $25.0^{\circ} \mathrm{C}(\Theta)$} \\
\hline PIB660 & $6.63 \times 10^{6}\left(6.49 \times 10^{6}\right)$ & & $8.0_{0}$ & 6.15 \\
\hline $\begin{array}{l}{ }^{\mathrm{a}} \text { The val } \\
\text { been reproc } \\
\text { mined from } \\
\text { from ref } 10\end{array}$ & $\begin{array}{l}\text { w and }\left\langle S^{2}\right\rangle^{1 / 2} \text { for AMS32 } \\
\mathrm{m} \text { refs } 9,10 \text {, and } 21 .{ }^{b} \mathrm{Th} \\
\text { easurements for a-P } \alpha \mathrm{MS} \\
\text { PIB in } n \text {-heptane at } 25.0\end{array}$ & $\begin{array}{l}\text { AMS55 } \\
\text { s of } M_{\mathrm{w}} \\
\text { ene at } 2\end{array}$ & $\begin{array}{l}\text { also of } R_{\mathrm{H}} \text { fo } \\
\text { entheses repre } \\
\text { which have }\end{array}$ & $\begin{array}{l}320 \text { have } \\
\text { ose deter- } \\
\text { eproduced }\end{array}$ \\
\hline
\end{tabular}

ples prepared by living anionic polymerization. We note that the initiating chain end of each a-P $\alpha$ MS sample is a sec-butyl group and the other end is a hydrogen atom. The values of the fraction of racemic diads $f_{\mathrm{r}}$ are 0.73 and 0.70 for AMS320 and AMS550, respectively. ${ }^{10,14}$ The one PIB sample PIB660 used is a fraction newly separated from the commercial sample of Aldrich Co., named 18,149-8, by the $\Theta$ column elution method ${ }^{15,16}$ with benzene as a solvent followed by fractional precipitation with benzene as a solvent and methanol as a precipitant.

The solvent cyclohexane for a-P $\alpha$ MS was purified according to a standard procedure. The solvent isoam$\mathrm{yl}$ isovalerate (IAIV) for PIB was purified by distillation under reduced pressure after dehydration with potassium carbonate.

\section{Static Light Scattering}

Static light scattering (SLS) measurements were carried out to determine the weight-average molecular weight $M_{\mathrm{w}}$ and $\left\langle S^{2}\right\rangle$ for the PIB sample PIB660 in IAIV at $25.0^{\circ} \mathrm{C}(\Theta)$ and also its $M_{\mathrm{w}}$ in $n$-heptane at $25.0^{\circ} \mathrm{C}$. A Fica 50 light-scattering photometer was used for all the measurements with vertically polarized incident light of wavelength $436 \mathrm{~nm}$. For a calibration of the apparatus, the intensity of light scattered from pure benzene was measured at $25.0^{\circ} \mathrm{C}$ at a scattering angle of $90^{\circ}$, where the Rayleigh ratio $R_{\mathrm{Uu}}\left(90^{\circ}\right)$ of pure benzene was taken as $46.5 \times 10^{-6} \mathrm{~cm}^{-1} .{ }^{17}$ The depolarization ratio $\rho_{\mathrm{u}}$ of pure benzene at 25.0 ${ }^{\circ} \mathrm{C}$ was found to be $0.41 \pm 0.01$. Scattered intensities were measured at six different concentrations and at scattering angles $\theta$ ranging from 17.5 to $55^{\circ}$. All the data obtained were analyzed by the Berry square-root plot. ${ }^{18}$

The most concentrated solutions of PIB660 in IAIV and in $n$-heptane were prepared gravimetrically and then made homogeneous by continuous stirring at ca. $50^{\circ} \mathrm{C}$ for $7 \mathrm{~d}$ and $3 \mathrm{~d}$, respectively. They were optically purified by filtration through a Teflon membrane of pore size $1.0 \mu \mathrm{m}$. The solutions of lower con- centrations were obtained by successive dilution, adding the solvents optically purified by filtration through a Teflon membrane of pore size $0.1 \mu \mathrm{m}$. The weight concentrations of the test solutions were converted to the polymer mass concentrations $c$ by the use of the densities of the solutions.

The values of $M_{\mathrm{w}}$ and $\left\langle S^{2}\right\rangle^{1 / 2}$ so determined for PIB660 are given in Table I along with those for AMS320 and AMS550 determined previously ${ }^{9,10}$ in cyclohexane at $30.5^{\circ} \mathrm{C}(\Theta)$ and also in toluene at $25.0^{\circ} \mathrm{C}$. For each sample, the values of $M_{\mathrm{w}}$ with and without parentheses represent the values in its good and $\Theta$ solvents, respectively, and are seen to be in good agreement with each other within experimental error. We note that the ratio of $M_{\mathrm{w}}$ to the numberaverage molecular weight $M_{\mathrm{n}}$ could be determined from analytical gel permeation chromatography (GPC) only for AMS320, its value being given in the third column of Table I. Although the values of $M_{\mathrm{w}} / M_{\mathrm{n}}$ for the other samples could not be determined with sufficient accuracy because of the lack of the GPC calibration curve in the necessary range of $M_{\mathrm{w}}$, their molecular weight distributions may be considered as narrow $\left(M_{\mathrm{w}} / M_{\mathrm{n}} \lesssim 1.1\right)$ as that of AMS320.

\section{Dynamic Light Scattering}

Dynamic light scattering (DLS) measurements were carried out to determine the translational diffusion coefficient $D$ for AMS550 in cyclohexane at $30.5^{\circ} \mathrm{C}(\Theta)$ and for PIB660 in IAIV at $25.0^{\circ} \mathrm{C}(\Theta)$. Measurements were also carried out to determine $\Omega(k)$ for all the a$\mathrm{P} \alpha \mathrm{MS}$ and PIB samples in the respective $\Theta$ solvents. For all the measurements, a Brookhaven Instruments model BI-200SM light scattering goniometer was used with vertically polarized incident light of wavelength $\lambda_{0}=488 \mathrm{~nm}$ from a Spectra-Physics model 2020 argon ion laser equipped with a model 583 temperature stabilized etalon for a single-frequency-mode operation. The photomultiplier tube used was EMI 9893B/350, the output from which was processed by a Brookhaven Instruments model BI-9000AT Digital 
Correlator. (An electric shutter was attached to the original detector alignment in order to monitor the dark count automatically.) The method of data analysis for $D$ is the same as that described in a previous paper. ${ }^{19}$ As for $\Omega(k)$, the CONTIN method ${ }^{20}$ was employed as before. ${ }^{1,2}$

For the determination of $D$ for each sample, the normalized autocorrelation function $g^{(2)}(t)$ of the scattered light intensity was measured at five concentrations and at $\theta$ ranging from 18 to $26^{\circ}$. For the determination of $\Omega(k)$, it was measured at $30,45,60,90,120$, and $150^{\circ}$ for all the samples. These measurements for each sample were carried out at the same concentrations as those in the case of the determination of $D$. The sampling times adopted for the determination of $D$ were $100 \mu$ s for AMS550 and $100-200 \mu$ s for PIB660. For the determination of $\Omega(k)$, two measurements with different sampling times were carried out for each solution at each angle in order to cover a wide range of time and to check its possible dependence on the choice of the sampling time. The sampling times so adopted were $0.5-20 \mu$ s for AMS320 and AMS550 and 1.0-100 $\mu$ s for PIB660.

The most concentrated solutions of AMS320 and AMS550 in cyclohexane were prepared gravimetrically and made homogeneous by continuous stirring at ca. $50^{\circ} \mathrm{C}$ for $7 \mathrm{~d}$. The test solutions of AMS320 and AMS550 and also of PIB660 were prepared in the manner mentioned in the last subsection. The values of the refractive index at $488 \mathrm{~nm}$ and of $\eta_{0}$ used are 1.426 and $0.818 \mathrm{cP}$, respectively, for cyclohexane at $30.5^{\circ} \mathrm{C}$ and 1.415 and $1.33 \mathrm{cP}$, respectively, for IAIV at $25.0^{\circ} \mathrm{C}$.

\section{RESULTS}

\section{Translational Diffusion Coefficient}

The values of $D$ determined in this study are $5.7_{4} \times$ $10^{-8} \mathrm{~cm}^{2} / \mathrm{s}$ for the a-P $\alpha$ MS sample AMS550 in cyclohexane at $30.5^{\circ} \mathrm{C}(\Theta)$ and $2.67 \times 10^{-8} \mathrm{~cm}^{2} / \mathrm{s}$ for the PIB sample PIB660 in IAIV at $25.0^{\circ} \mathrm{C}(\Theta)$. The values of the hydrodynamic radius $R_{\mathrm{H}}$ calculated from the defining equation

$$
R_{\mathrm{H}}=k_{\mathrm{B}} T / 6 \pi \eta_{0} D
$$

with the above values of $D$ are given in the fifth column of Table I along with that previously determined for AMS320 in cyclohexane at $30.5^{\circ} \mathrm{C} .{ }^{21}$

The ratio $\rho=\left\langle S^{2}\right\rangle^{1 / 2} / R_{\mathrm{H}}$ may be evaluated to be 1.29 and 1.30 for AMS550 and PIB660, respectively, using the values of $\left\langle S^{2}\right\rangle^{1 / 2}$ and $R_{\mathrm{H}}$ given in the fourth and fifth columns, respectively, of Table I. The value 1.29 of $\rho$ for AMS550 agrees well with the previous ones $^{21} 1.26-1.29$ determined for a-P $\alpha \mathrm{MS}$ in the range of $4 \times 10^{5} \lesssim M_{\mathrm{w}} \lesssim 3 \times 10^{6}$, including AMS320, and the value 1.30 for PIB660 also with the previous ones $^{19} 1.29-1.33$ determined for PIB in the range of $4 \times 10^{5} \lesssim M_{\mathrm{w}} \lesssim 2 \times 10^{6}$. It may then be concluded that both the present SLS and/or DLS measurements for the samples AMS550 and PIB660 with rather large $M_{\mathrm{w}}$ could be carried out successfully.

\section{First Cumulant}

The first cumulant $\Omega(k)$ (as a function of the magnitude $k$ of the scattering vector) defined as the initial decay rate of the dynamic structure factor $S(k, t)$ (as a function of $k$ and time $t)$, i.e., $\Omega(k) \equiv-[d \ln S(k, t) /$ $d t]_{t=0}$ may be written in terms of the normalized autocorrelation function $g^{(2)}(t)$ of the scattered light intensity observed in DLS measurements as follows,

$$
\Omega(k)=-\left[\frac{\mathrm{d}}{\mathrm{d} t}\left\{\frac{1}{2} \ln \left[g^{(2)}(t)-1\right]\right\}\right]_{t=0}
$$

We note that $k$ is related to the scattering angle $\theta$ by

$$
k=(4 \pi / \tilde{\lambda}) \sin (\theta / 2)
$$

with $\tilde{\lambda}$ the wavelength of the incident light in the solvent.

As in the case of the previous studies of $\Omega$ for flexible and semiflexible polymers, ${ }^{1,2}$ the initial tangent of $(1 / 2) \ln \left[g^{(2)}(t)-1\right]$ at each $\theta$ for each test solution has been determined by extrapolating the data to $t=0$ by the use of the Fortran program package CONTIN ${ }^{20}$ supplied by Brookhaven Instruments. Then the values of the initial slope so determined at finite concentrations at each $\theta$ for each sample have been linearly extrapolated to $c=0$ to determine $\Omega$ at infinite dilution. In Table II are given the values of $\Omega(k)$ and $\eta_{0} \Omega(k) /$ $k_{\mathrm{B}} T k^{3}$ so determined.

\section{DISCUSSION}

\section{Nonuniversality_Effects of Chain Stiffness and Local Chain Conformation}

Figure 1 shows plots of $\eta_{0} \Omega(k) / k_{\mathrm{B}} T k^{3}$ against the reduced magnitude $\bar{k}$ of the scattering vector defined by

$$
\bar{k} \equiv\left\langle S^{2}\right\rangle^{1 / 2} k
$$

i.e., the so-called universal plot. The unfilled circles with pip up and down represent the present experimental values for the a-P $\alpha$ MS samples AMS320 and AMS550, respectively, in cyclohexane at $30.5^{\circ} \mathrm{C}(\Theta)$, and the filled circles those for the PIB sample PIB660 in IAIV at $25.0^{\circ} \mathrm{C}(\Theta)$. For comparison, there are also shown the previous results for a-PS ${ }^{1}$ with $M_{\mathrm{w}}=$ $6.40 \times 10^{6}$ (square with pip up) and $8.04 \times 10^{6}$ (square with pip down) in cyclohexane at $34.5^{\circ} \mathrm{C}$ $(\Theta)$ and for PHIC ${ }^{2}$ with $M_{\mathrm{w}}=1.04 \times 10^{5}$ (unfilled triangle), $3.63 \times 10^{5}$ (filled triangle), and $7.71 \times 10^{5}$ 
(unfilled inverted triangle) in $n$-hexane at $25.0^{\circ} \mathrm{C}$. In the figure the heavy curves represent the HW theoretical values, and the light ones the theoretical values for the Kratky-Porod (KP) wormlike chain model. ${ }^{3}$ They are discussed in the next subsection.

Table II. Values of $\Omega(k)$ and $\eta_{0} \Omega(k) / k_{\mathrm{B}} T k^{3}$ for poly $\left(\alpha\right.$-methylstyrene) in cyclohexane at $30.5^{\circ} \mathrm{C}(\Theta)$ and Polyisobutylene in IAIV at $25.0^{\circ} \mathrm{C}(\Theta)$

\begin{tabular}{lcccc}
\hline Sample & $\begin{array}{c}\theta \\
(\mathrm{deg})\end{array}$ & $\begin{array}{c}10^{-3} k \\
\left(\AA^{-1}\right)\end{array}$ & $\begin{array}{c}\Omega(k) \\
\left(\mathrm{s}^{-1}\right)\end{array}$ & $\eta_{0} \Omega(k) / k_{\mathrm{B}} T k^{3}$ \\
\hline AMS320 & 30 & 0.950 & $7.0_{3} \times 10^{2}$ & 0.160 \\
& 45 & 1.405 & $1.5_{6} \times 10^{3}$ & 0.110 \\
& 60 & 1.836 & $2.7_{8} \times 10^{3}$ & $0.087_{6}$ \\
& 90 & 2.596 & $6.2_{4} \times 10^{3}$ & $0.069_{6}$ \\
& 120 & 3.179 & $1.0_{1} \times 10^{4}$ & $0.061_{1}$ \\
& 150 & 3.546 & $1.3_{0} \times 10^{4}$ & $0.056_{8}$ \\
& & & & \\
AMS550 & 30 & 0.950 & $5.5_{3} \times 10^{2}$ & 0.126 \\
& 45 & 1.405 & $1.3_{0} \times 10^{3}$ & $0.091_{2}$ \\
& 60 & 1.836 & $2.3_{4} \times 10^{3}$ & $0.073_{7}$ \\
& 90 & 2.596 & $5.1_{1} \times 10^{3}$ & $0.057_{0}$ \\
& 120 & 3.179 & $8.4_{8} \times 10^{3}$ & $0.051_{5}$ \\
& 150 & 3.546 & $1.1_{4} \times 10^{4}$ & $0.050_{0}$ \\
& & & & \\
& 30 & 0.943 & $2.6_{3} \times 10^{2}$ & 0.102 \\
& 45 & 1.394 & $6.1_{0} \times 10^{2}$ & $0.072_{8}$ \\
& 60 & 1.822 & $1.1_{6} \times 10^{3}$ & $0.061_{9}$ \\
& 90 & 2.576 & $2.7_{8} \times 10^{3}$ & $0.052_{5}$ \\
& 120 & 3.156 & $4.8_{8} \times 10^{3}$ & $0.050_{2}$ \\
& 150 & 3.520 & $6.6_{1} \times 10^{3}$ & $0.049_{0}$ \\
\hline & & & &
\end{tabular}

It is seen that there are slight but appreciable differences in the height of the $k^{3}$-region (the plateau region) in the plot between the three flexible polymers, and moreover, that the order of the height (a-P $\alpha \mathrm{MS}<$ PIB $<$ a-PS) is not correlated with that of the magnitude of $\lambda^{-1}$ [PIB $(15.3 \AA)<$ a-PS $(20.6 \AA)<$ a-P $\alpha$ MS $(46.8 \AA)]$. Such differences may then be regarded as arising from that in local chain conformation as well as in chain stiffness. As for PHIC, whose $\lambda^{-1}$ value $\left(840 \AA\right.$ in $n$-hexane ${ }^{22}$ ) is one order of magnitude larger than those for the three flexible polymers, the plots deviate appreciably upward from those for the latter three. Further, the plots for the three PHIC samples deviate appreciably from each other, while those for the two samples of each of a-P $\alpha$ MS and a-PS form a single-composite curve within experimental error. Such characteristics of the plots for PHIC may be considered to arise from very large chain stiffness. The present experimental results seem to confirm the previous conclusion that the universality in the $k^{3}$-region cannot be realized because of the effects of chain stiffness and local chain conformation. In the next subsection, we further discuss this difference in the behavior of the plot on the basis of the HW (and KP) theory.

\section{Comparison with the HW Theory}

We begin by giving a short sketch of the (discrete) dynamic HW chain model (and also of the KP chain as its special case). ${ }^{3}$ It is composed of $N$ identical rigid subbodies joined successively with bonds of fixed

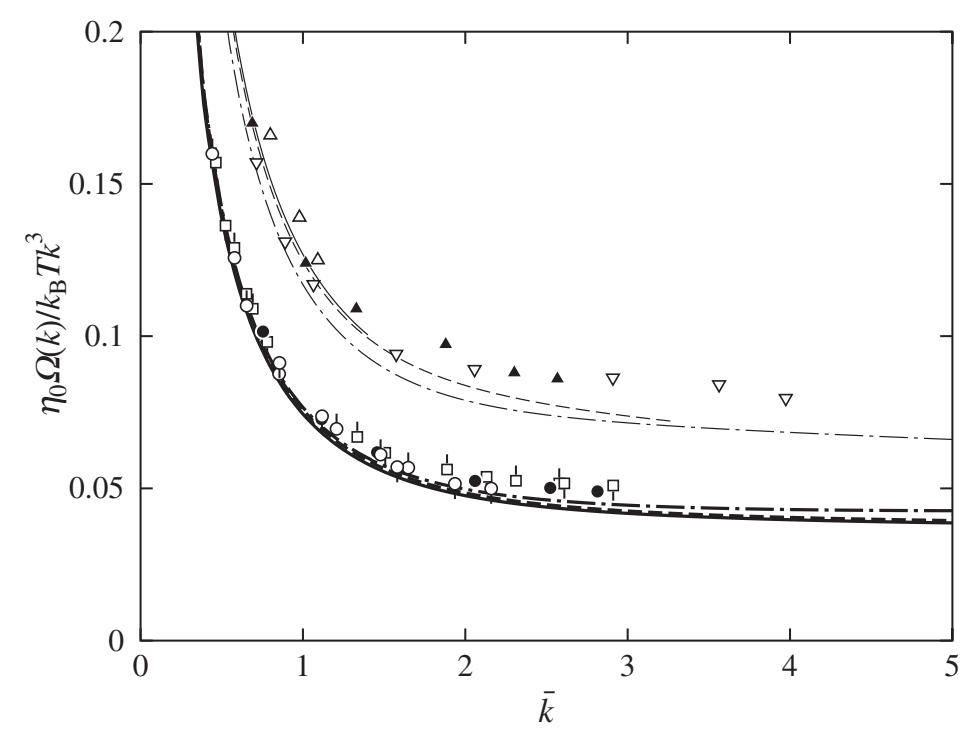

Figure 1. Plots of $\eta_{0} \Omega(k) / k_{\mathrm{B}} T k^{3}$ against the reduced magnitude $\bar{k}$ of the scattering vector. The unfilled circles with pip up and down represent the present experimental values for the a-P $\alpha$ MS samples AMS320 and AMS550, respectively, in cyclohexane at $30.5^{\circ} \mathrm{C}(\Theta)$, and the filled circles those for the PIB sample PIB660 in IAIV at $25.0^{\circ} \mathrm{C}(\Theta)$. Also are shown the results for a-PS ${ }^{1}$ with $M_{\mathrm{w}}=6.40 \times 10^{6}$ (square with pip up) and $8.04 \times 10^{6}$ (square with pip down) in cyclohexane at $34.5^{\circ} \mathrm{C}(\Theta)$ and for PHIC ${ }^{2}$ with $M_{\mathrm{w}}=1.04 \times 10^{5}$ (unfilled triangle), $3.63 \times 10^{5}$ (filled triangle), and $7.71 \times 10^{5}$ (unfilled inverted triangle) in $n$-hexane at $25.0^{\circ} \mathrm{C}$. The heavy solid, dashed, and dotdashed curves represent the HW theoretical values for a-P $\alpha$ MS, PIB, and a-PS, respectively, and the light solid, dashed, and dot-dashed curves represent the KP theoretical values for the PHIC samples with the smallest through largest $M_{\mathrm{w}}$, respectively (see the text). 
length $a$, where their centers are located nearly on the contour of the continuous HW chain of length $L$ and each subbody has translational and rotatory friction coefficients $\zeta_{\mathrm{t}}$ and $\zeta_{\mathrm{r}}$. Specifically, the pair potential between successive two subbodies is chosen so that the static behavior of the dynamic model becomes identical with that of the continuous HW chain. ${ }^{3}$ The latter is an elastic wire model with both bending and torsional energies. In particular, for flexible chains, the bending and torsional force constants associated with the two kinds of elastic energies may be set equal to each other. The HW chain may then be described in terms of the static stiffness parameter $\lambda^{-1}$ (equal to the bending force constant multiplied by $\left.2 / k_{\mathrm{B}} T\right)$ and the constant differential-geometrical curvature $\kappa_{0}$ and torsion $\tau_{0}$ of the characteristic helix, i.e., the regular helical form taken by the contour of the chain at the minimum zero of its total elastic energy along with the shift factor $M_{\mathrm{L}}$ as defined as the molecular weight per unit contour length. A special case of the HW chain with $\kappa_{0}=0$ but with the two (different) force constants is the generalized KP chain, which is the model for most of stiff (or semiflexible) chains (with large $\lambda^{-1}$ ). If the torsion of the chain is not considered, the generalized KP chain is identical with the original KP chain (with $\kappa_{0}=0$ and with vanishing torsional force constant) and the parameter $\tau_{0}$ does not appear. In what follows, therefore, both the original and generalized KP chains are referred to simply as the KP chain. The number of subbodies $N$ in the discrete model is related to $L$ by $L=N \Delta s$, where $\Delta s$ is the contour length per subbody and is uniquely related to $a$. (In the case of flexible polymers, the subbody is chosen as a repeat unit of a given polymer chain.)

Now, for the dynamic HW chain (without excluded volume) such that each subbody has an isotropic scatterer, the dimensionless quantity $\eta_{0} \Omega(k) / k_{\mathrm{B}} T k^{3}$ may be written in the form 3,4

$$
\eta_{0} \Omega(k) / k_{\mathrm{B}} T k^{3}=(1 / 6 \pi)[\rho / \bar{k}+F(\bar{k}) / \bar{k}]
$$

where $F(\bar{k})$, which is given by eq 10.66 of ref 3 , may be expressed in terms of the solutions of the eigenvalue problem associated with the diffusion operator appearing in the coarse-grained version of the diffusion equation, ${ }^{3}$ along with $\left\langle S^{2}\right\rangle$ and the mean-square endto-end distance of the HW chain, which are given by eqs 4.83 and 4.82 , respectively, of ref 3 . The dimensionless quantity $F(\bar{k})$ as a function of $\bar{k}$ depends on the dimensionless parameters $\lambda^{-1} \kappa_{0}, \lambda^{-1} \tau_{0}, \lambda \Delta s$ (or $\lambda a$ ), $N, r_{1}=\zeta_{\mathrm{t}} / 3 \pi \eta_{0} a$, and $r_{2}=\zeta_{\mathrm{r}} / a^{2} \zeta_{\mathrm{t}}$ and may be evaluated numerically.

In Figure 1, the heavy solid and dashed curves represent the HW theoretical values calculated from eq 5 for a-P $\alpha \mathrm{MS}$ and PIB, respectively. In the calculation we have used the parameter values $\lambda^{-1} \kappa_{0}=3.0$, $\lambda^{-1} \tau_{0}=0.9$, and $\lambda^{-1}=46.8 \AA$ for a-P $\alpha \mathrm{MS}^{9}$ and $\lambda^{-1} \kappa_{0}=1.0, \lambda^{-1} \tau_{0}=0$, and $\lambda^{-1}=15.3 \AA$ for PIB ${ }^{8}$ which have been determined experimentally from an analysis of the $M_{\mathrm{w}}$ dependence of the unperturbed or perturbed $\left\langle S^{2}\right\rangle$. The subbody corresponds to a repeat unit of a given polymer chain, as mentioned above, and then the values of $\lambda \Delta s$ have been taken to be 0.0634 and 0.175 for a-P $\alpha$ MS and PIB, respectively, which have been calculated using the relation $\Delta s=$ $M_{0} / M_{\mathrm{L}}$ with the respective values 118 and 56 of the molecular weight $M_{0}$ of the repeat unit and the respective values 39.8 and $20.9 \AA^{-1}$ of $M_{\mathrm{L}}$ for a-P $\alpha \mathrm{MS}^{9}$ and PIB. ${ }^{8}$ Further, we have chosen $r_{1}$ and $r_{2}$ to be 1 and 10 , respectively, which may be of reasonable order, and $N+1$ to be $10^{4}$, which corresponds to the limit of the capacity of the computer used. As for $\rho$, we have used the present experimental values $1.27_{5}$ and 1.30 for a-P $\alpha \mathrm{MS}$ and PIB, respectively, the former value being the mean of values obtained for the two a-P $\alpha$ MS samples AMS320 and AMS550.

In Figure 1 are also shown the HW theoretical values for the a-PS (heavy dot-dashed curve) and the KP theoretical values for the three PHIC samples (light solid, dashed, dot-dashed curves for the samples with the smallest through largest $M_{\mathrm{w}}$, respectively), which have been reproduced from Figure 2 of ref 1 and from Figure 3 of ref 2, respectively.

It is seen from the figure that the HW (and KP) theory may correctly predict the order of the height of the plateau in the $k^{3}$-region for the three flexible polymers (a-P $\alpha \mathrm{MS}<\mathrm{PIB}<\mathrm{a}$-PS) and also the dependence of $\eta_{0} \Omega(k) / k_{\mathrm{B}} T k^{3}$ on $M_{\mathrm{w}}$ for the PHIC, although all the theoretical curves deviate somewhat downward from the corresponding data points and agreement between theory and experiment is not completely quantitative. The qualitative agreement between theory and experiment for the three flexible polymers indicates that there is very little doubt that the height of the $k^{3}$-region for flexible polymers with very large $M_{\mathrm{w}}$ depends slightly on both chain stiffness and local chain conformation and therefore that the universality cannot in general be realized. For semiflexible polymers, whose conformational behavior cannot be described by the Gaussian chain statistics, the height of $\eta_{0} \Omega(k) / k_{\mathrm{B}} T k^{3}$ is much larger than that for flexible polymers in the large $k$ region, as predicted by the theory.

\section{CONCLUSIONS}

The first cumulant $\Omega(k)$ of the dynamic structure factor as a function of the magnitude $k$ of the scattering vector has been determined for the two a-P $\alpha \mathrm{MS}$ samples in cyclohexane at $30.5^{\circ} \mathrm{C}(\Theta)$ and the one 
PIB sample in IAIV at $25.0^{\circ} \mathrm{C}(\Theta)$. From a comparison of the present data for a-P $\alpha$ MS and PIB along with the previous ones for a-PS and PHIC with the HW (and KP) theory, we have reconfirmed the conclusion that the universality predicted by the Gaussian chain theory for the plot of $\eta_{0} \Omega(k) / k_{\mathrm{B}} T k^{3}$ against $\left\langle S^{2}\right\rangle^{1 / 2} k$ cannot be realized for flexible polymers even with very high molecular weights $\left(\simeq 10^{6}-10^{7}\right)$, and that the height of the plateau in the $k^{3}$-region depends on chain stiffness and local chain conformation.

Acknowledgment. This research was supported in part by the 21 st century COE program " $\mathrm{COE}$ for a United Approach to New Materials Science" from the Ministry of Education, Culture, Sports, Science and Technology, Japan.

\section{REFERENCES}

1. N. Sawatari, T. Yoshizaki, and H. Yamakawa, Macromolecules, 31, 4218 (1998).

2. N. Yoshida, T. Yoshizaki, and H. Yamakawa, Macromolecules, 33, 3254 (2000).

3. H. Yamakawa, "Helical Wormlike Chains in Polymer Solutions,” Springer, Berlin, 1997.

4. T. Yoshizaki, M. Osa, and H. Yamakawa, J. Chem. Phys., 106, 2828 (1997).

5. P. G. de Gennes, Physics, 3, 37 (1967).

6. E. Dubois-Violette and P. G. de Gennes, Physics, 3, 181
(1967).

7. Z. Akcasu and H. Gurol, J. Polym. Sci., Polym. Phys. Ed., 14, 1 (1976).

8. M. Yamada, M. Osa, T. Yoshizaki, and H. Yamakawa, Macromolecules, 30, 7166 (1997).

9. M. Osa, T. Yoshizaki, and H. Yamakawa, Macromolecules, 33, 4828 (2000), and succeeding papers.

10. M. Osa, Y. Ueno, T. Yoshizaki, and H. Yamakawa, Macromolecules, 34, 6402 (2001).

11. Y. Tominaga, I. Suda, M. Osa, T. Yoshizaki, and H. Yamakawa, Macromolecules, 35, 1381 (2002).

12. W. Tokuhara, M. Osa, T. Yoshizaki, and H. Yamakawa, Macromolecules, 36, 5311 (2003).

13. M. Osa, T. Yoshizaki, and H. Yamakawa, Polym. J., 36, 634 (2004).

14. M. Osa, M. Sumida, T. Yoshizaki, H. Yamakawa, K. Ute, T. Kitayama, and K. Hatada, Polym. J., 32, 361 (2000).

15. T. Matsumoto, N. Nishioka, and H. Fujita, J. Polym. Sci., Polym. Phys. Ed., 10, 23 (1972).

16. Y. Nakamura, K. Akasaka, K. Katayama, T. Norisuye, and A. Teramoto, Macromolecules, 25, 1134 (1992).

17. Gj. Deželić and J. Vavra, Croat. Chem. Acta, 38, 35 (1966).

18. G. C. Berry, J. Chem. Phys., 44, 4550 (1966).

19. T. Konishi, T. Yoshizaki, and H. Yamakawa, Macromolecules, 24, 5614 (1991).

20. S. W. Provencher, Comput. Phys. Commun., 27, 213 (1982).

21. I. Suda, Y. Tominaga, M. Osa, T. Yoshizaki, and H. Yamakawa, Macromolecules, 33, 9322 (2000).

22. H. Murakami, T. Norisuye, and H. Fujita, Macromolecules, 13, 345 (1980). 